\title{
Mode of action and functional significance of $7 \alpha$-hydroxypregnenolone stimulating locomotor activity
}

\author{
Shogo Haraguchi ${ }^{1}$, Masahiro Matsunaga ${ }^{1}$, Hubert Vaudry ${ }^{2}$ and Kazuyoshi Tsutsui ${ }^{1}$ * \\ ${ }^{1}$ Laboratory of Integrative Brain Sciences, Department of Biology, Waseda University, and Center for Medical Life Science of Waseda University, Tokyo, Japan \\ ${ }^{2}$ Laboratory of Neuronal and Neuroendocrine Differentiation and Communication (INSERM U982), European Institute for Peptide Research, University of Rouen, \\ Mont-Saint-Aignan, France
}

\section{Edited by:}

Eric W. Roubos, Radboud University

Nijmegen, Netherlands

\section{Reviewed by:}

Gilberto Mosconi, University of

Camerino, Italy

H. Bobby Fokidis, University of British

Columbia, Canada

*Correspondence:

Kazuyoshi Tsutsui, Laboratory of Integrative Brain Sciences,

Department of Biology, Waseda

University, and Center for Medical

Life Science of Waseda University,

2-2 Wakamatsu-cho, Shinjuku-ku,

Tokyo 162-8480, Japan.

e-mail: k-tsutsui@waseda.jp
Previous studies over the past two decades have demonstrated that the brain and other nervous systems possess key steroidogenic enzymes and produces pregnenolone and other various neurosteroids in vertebrates in general. Recently, $7 \alpha$-hydroxypregnenolone, a novel bioactive neurosteroid, was identified in the brain of newts and quail. Importantly, this novel neurosteroid is produced from pregnenolone through the enzymatic activity of cytochrome $\mathrm{P}^{4} \mathrm{~S}_{7 \alpha}$ and acts on brain tissue as a neuronal modulator to stimulate locomotor activity in these vertebrates. Subsequently, the mode of action of $7 \alpha-$ hydroxypregnenolone was demonstrated. $7 \alpha$-Hydroxypregnenolone stimulates locomotor activity through activation of the dopaminergic system. To understand the functional significance of $7 \alpha$-hydroxypregnenolone in the regulation of locomotor activity, diurnal, and seasonal changes in $7 \alpha$-hydroxypregnenolone synthesis were further characterized. Melatonin derived from the pineal gland and eyes regulates $7 \alpha$-hydroxypregnenolone synthesis in the brain, thus inducing diurnal locomotor changes. Prolactin, an adenohypophyseal hormone, regulates $7 \alpha$-hydroxypregnenolone synthesis in the brain, and also induces seasonal locomotor changes. In addition, 7a-hydroxypregnenolone mediates corticosterone action to modulate locomotor activity under stress. This review summarizes the current knowledge regarding the mode of action and functional significance of $7 \alpha$-hydroxypregnenolone, a newly identified bioactive neurosteroid stimulating locomotor activity.

Keywords: neurosteroids, $7 \alpha$-hydroxypregnenolone, dopamine, melatonin, prolactin, locomotor activity, diurnal and seasonal changes, stress

\section{INTRODUCTION}

The brain has traditionally been considered as a target site for peripheral steroid hormones. In addition to this classical concept, it is now established that steroids can be synthesized de novo in the central and peripheral nervous systems. Such steroids are called "neurosteroids." De novo neurosteroidogenesis in the brain from cholesterol is considered to be a conserved property across vertebrates, (for reviews, see Baulieu, 1997; Tsutsui et al., 1999, 2000; Compagnone and Mellon, 2000; Mellon and Vaudry, 2001; Tsutsui et al., 2003, 2006; Tsutsui and Mellon, 2006; Do-Rego et al., 2009).

Seasonally breeding wild animals, such as amphibians, have served as excellent animal models to investigate the biosynthesis and biological actions of neurosteroids. Previous studies over the past two decades have demonstrated that the brain of amphibians possesses several key steroidogenic enzymes and produces pregnenolone, a precursor of steroid hormones (Takase et al., 1999, 2011; Inai et al., 2003), and other various neurosteroids (MensahNyagan et al., 1994, 1996a,b, 1999; Beaujean et al., 1999; Takase et al., 2002; Inai et al., 2003; Matsunaga et al., 2004; Do-Rego et al., 2007; Bruzzone et al., 2010). The formation of neurosteroids from cholesterol is now documented in amphibians as in other vertebrates.

However, the biosynthetic pathways leading to the formation of neurosteroids in vertebrate brains was not fully characterized (for a review, see Tsutsui et al., 2006). In fact, Matsunaga et al. (2004) recently found that the brain of newts actively produces $7 \alpha$-hydroxypregnenolone, a previously undescribed amphibian neurosteroid, from pregnenolone. Importantly, $7 \alpha-$ hydroxypregnenolone acts on brain tissue as a novel neuronal modulator to stimulate locomotor activity of newts (Matsunaga et al., 2004). Tsutsui et al. (2008) also identified $7 \alpha-$ and $7 \beta$ hydroxypregnenolone in the brain of quail by using biochemical techniques (Tsutsui et al., 2008). $7 \alpha$-Hydroxypregnenolone, but not $7 \beta$-hydroxypregnenolone, stimulates locomotor activity in quail (Tsutsui et al., 2008) as in newts (Matsunaga et al., 2004). It was further found that cytochrome $\mathrm{P} 450_{7 \alpha}$ catalyzes the conversion of pregnenolone to $7 \alpha$-hydroxypregnenolone in the brain of these vertebrates (Tsutsui et al., 2008; Haraguchi et al., 2010).

Based on these recent findings, this review summarizes the current knowledge regarding the mode of action and functional significance of $7 \alpha$-hydroxypregnenolone, a new key regulator of locomotor activity in vertebrates.

\section{OUTLINE OF 7 $\alpha$-HYDROXYPREGNENOLONE AND ITS BIOLOGICAL ACTION} IDENTIFICATION OF $7 \alpha$-HYDROXYPREGNENOLONE IN THE BRAIN

Our preliminary study initially suggested that the brain of newts actively produces an unknown amphibian neurosteroid from 
pregnenolone. Subsequently, Matsunaga et al. (2004) demonstrated that this unknown pregnenolone metabolite is $7 \alpha-$ hydroxypregnenolone in the newt brain (Figure 1), based on biochemical techniques combined with high-performance liquid chromatography (HPLC), thin-layer chromatography (TLC), and gas chromatography-mass spectrometry (GC-MS) analyses. Tsutsui et al. (2008) further demonstrated that the quail brain also produces $7 \alpha$ - and $7 \beta$-hydroxypregnenolone by using the same biochemical techniques (Figure 1).

\section{IDENTIFICATION OF CYTOCHROME P450 ${ }_{7 \alpha}$ IN THE BRAIN}

$7 \alpha$-Hydroxypregnenolone is considered to be synthesized from pregnenolone through the enzymatic activity of cytochrome $\mathrm{P}_{450}{ }_{7 \alpha}$ (Figure 1). Haraguchi et al. (2010) identified a cDNA encoding a putative cytochrome $\mathrm{P}^{4} 50_{7 \alpha}$ from the newt brain. The newt P450 $7 \alpha$ cDNA had a full length of $2598 \mathrm{bp}$. The enzymatic activity of this putative newt $\mathrm{P} 450_{7 \alpha}$ was then demonstrated (Haraguchi et al., 2010). The homogenate of COS-7 cells transfected with the putative newt $\mathrm{P} 450_{7 \alpha}$ cDNA converted pregnenolone into $7 \alpha$-hydroxypregnenolone as shown by HPLC analysis, and the inhibitor of cytochrome P450s, ketoconazole, abolished this metabolic process. COS-7 cells without transfection of newt P450 $0_{\alpha}$ cDNA did not convert pregnenolone into $7 \alpha$ hydroxypregnenolone. $7 \alpha$-Hydroxypregnenolone synthesis was further confirmed by GC-MS analysis (Haraguchi et al., 2010).

A full length of $2341 \mathrm{bp}$ cDNA prepared from the quail brain was also identified as encoding a putative cytochrome $\mathrm{P}_{450} 0_{7 \alpha}$ (Tsutsui et al., 2008). The enzymatic activity of this putative quail P450 $7 \alpha$ was demonstrated in homogenates of COS-7 cells transfected with the putative quail $\mathrm{P} 450_{7 \alpha} \mathrm{CDNA}$ (Tsutsui et al., 2008). As demonstrated by HPLC and GC-MS analyses, the homogenate converted pregnenolone to $7 \alpha$-hydroxypregnenolone. Although it is still unclear whether cytochrome $\mathrm{P} 450_{7 \alpha}$ can also convert pregnenolone to $7 \beta$-hydroxypregnenolone, the presence of $7 \beta$-hydroxypregnenolone as well as $7 \alpha$-hydroxypregnenolone is evident in the quail brain (Tsutsui et al., 2008; Figure 1).

The production of $7 \alpha$-hydroxypregnenolone in the brain may be a conserved property of vertebrates, because this neurosteroid has also been identified in the brain of mammals (Akwa et al., 1992; Doostzadeh and Morfin, 1997; Weill-Engerer et al., 2003; Yau et al., 2003).

\section{BIOLOGICAL ACTION OF $7 \alpha$-HYDROXYPREGNENOLONE}

Because $7 \alpha$-hydroxypregnenolone is actively produced in the brain of newts, this seasonally breeding amphibian has served as a suitable animal model to investigate the biological action of $7 \alpha$-hydroxypregnenolone. $7 \alpha$-Hydroxypregnenolone synthesis in the brain of male newts showed marked changes during the annual breeding cycle, with a maximum level in the spring breeding period when locomotor activity of wild populations of the same species increases (Matsunaga et al., 2004). Matsunaga et al. (2004) therefore analyzed the effect of $7 \alpha$ hydroxypregnenolone on locomotor activity. For behavioral testing, newts were placed individually in a water-filled aquarium maintained at $18 \pm 2{ }^{\circ} \mathrm{C}$; each testing arena was marked with parallel lines to define four equal sectors (Matsunaga et al., 2004). Immediately after administration of $7 \alpha$-hydroxypregnenolone, locomotor activity was quantified by counting the total number of lines crossed during a 30-min observation (Matsunaga et al., 2004) according to a previous method (Moore and Miller, 1984; Lowry et al., 2001). Locomotion consisted of a combination of walking and swimming movements (Matsunaga et al., 2004). Administration of $7 \alpha$-hydroxypregnenolone acutely increases locomotor activity of male newts in the non-breeding period when endogenous $7 \alpha$-hydroxypregnenolone synthesis in the brain is low (Matsunaga et al., 2004). This stimulatory effect occurred in a dose-dependent manner with a threshold dose ranging from 0.5 to $1 \mathrm{ng}$ through intracerebroventricular (i.c.v.) injection, corresponding to the physiological range observed in the brain of normal newts (Matsunaga et al., 2004). Accordingly, $7 \alpha-$ hydroxypregnenolone may act as a novel neuronal modulator to stimulate locomotor activity of male newts, and the increase in locomotor activity of male newts that occurs during the spring breeding period may be ascribed to an increase in the production of $7 \alpha$-hydroxypregnenolone.

Because the quail displays a robust locomotor activity rhythm when held under typical light/dark lighting schemes (Wilson, 1972; Wada, 1979), this bird has also served as an appropriate animal model to investigate the biological action of $7 \alpha$ and $7 \beta$-hydroxypregnenolone. Both neurosteroids were therefore administered i.c.v. to male quail during night, when activity is low, to examine whether they affect locomotor activity (Tsutsui et al., 2008). For behavioral testing, quail were placed individually in an empty soundproof chamber (Tsutsui et al., 2008). For $30 \mathrm{~min}$ after administration of $7 \alpha$-hydroxypregnenolone, locomotor activity was measured by using an implantable telemetry system (Tsutsui et al., 2008). A stimulatory dosedependent effect of $7 \alpha$-hydroxypregnenolone was also observed in male quail (Tsutsui et al., 2008). 7 $\beta$-Hydroxypregnenolone did not influence locomotor activity (Tsutsui et al., 2008). It

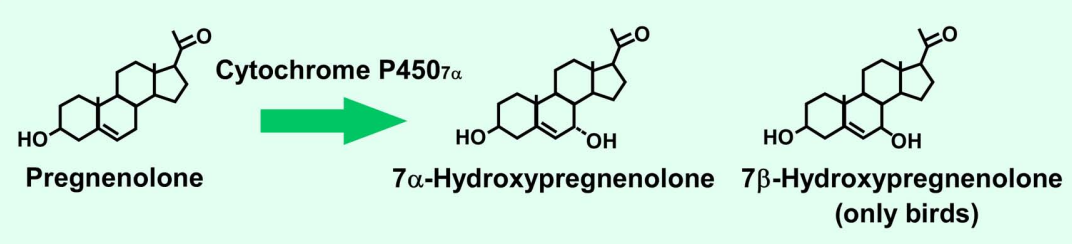

FIGURE 1 | $7 \alpha$-Hydroxypregnenolone synthesis by cytochrome $\mathrm{P450}_{7 \alpha}$ in the brain of newts and quail. See the text for details. 
thus appears that $7 \alpha$-hydroxypregnenolone acts as a neuronal modulator to stimulate locomotor activity in male quail as in male newts.

\section{MODE OF ACTION OF $7 \alpha$-HYDROXYPREGNENOLONE $7 \alpha$-HYDROXYPREGNENOLONE ACTION THROUGH DOPAMINERGIC SYSTEM}

To understand the mode of action of $7 \alpha$-hydroxypregnenolone on locomotion, Matsunaga et al. (2004) measured the concentrations of several monoamines by HPLC-electrochemical detection (ECD) $5 \mathrm{~min}$ after an i.c.v. injection of $7 \alpha$-hydroxypregnenolone to non-breeding male newts. $7 \alpha$-Hydroxypregnenolone significantly increased the concentration of dopamine in the male newt brain, particularly in the rostral brain region including the striatum, which is known to be involved in the regulation of locomotor behavior (Matsunaga et al., 2004). In contrast, there were no significant differences in the concentrations of other monoamines, i.e., norepinephrine, epinephrine, and 5-hydroxytryptamine (Matsunaga et al., 2004).

$7 \alpha$-Hydroxypregnenolone treatment resulted in a concentrationdependent increase in the release of dopamine from cultured male newt brain tissue with the threshold concentration ranged between $10^{-8}$ and $10^{-7} \mathrm{M}$ (Matsunaga et al., 2004). Furthermore, the effect of $7 \alpha$-hydroxypregnenolone on locomotion was abolished by administration of haloperidol or sulpiride, two dopamine $\mathrm{D}_{2}$ receptor antagonists, but not by administration of the dopamine $\mathrm{D}_{1}$ receptor antagonist SCH23390 (Matsunaga et al., 2004). Accordingly, it is considered that the stimulatory effect of $7 \alpha$ hydroxypregnenolone on locomotor activity is mediated through dopamine $\mathrm{D}_{2}$ receptors. To recapitulate, $7 \alpha$-hydroxypregnenolone synthesized actively in the diencephalon and rhombencephalon, by acting on dopaminergic neurons localized in the posterior tuberal nucleus (PT) and ventral tegmental area (VTA), may induce dopamine release from their terminals in the rostral brain region, notably in the striatum and nucleus accumbens (NA), and consequently increase locomotor activity of newts (Matsunaga et al., 2004; Figure 2).

In the male quail brain, the expression of cytochrome $\mathrm{P} 450_{7 \alpha}$ mRNA was localized in the nucleus preopticus medialis (POM), the nucleus paraventricularis magnocellularis (PVN), the nucleus ventromedialis hypothalami (VMN), the nucleus dorsolateralis anterior thalami (DLA), and the nucleus lateralis anterior thalami (LA; Tsutsui et al., 2008). In quail (Tsutsui et al., 2008) as in newts (Matsunaga et al., 2004), $7 \alpha$-hydroxypregnenolone increased the concentration of dopamine in the telencephalic region that encompasses the striatum (Sanberg, 1983; Sharp et al., 1987; Bardo et al., 1990). In birds, dopaminergic neurons that are located in the mesencephalic region, including the VTA and the substantia nigra $(\mathrm{SN})$, project to the telencephalon notably the striatum (Mezey and Csillag, 2002; Hara et al., 2007). Interestingly, the telencephalic region is enriched with dopamine $D_{1}$ and $\mathrm{D}_{2}$ receptors in birds (Ball et al., 1995; Levens et al., 2000). Accordingly, $7 \alpha$-hydroxypregnenolone synthesized actively in the diencephalon, by acting on dopamine neurons localized in the VTA and SN, may induce dopamine release from their termini in the striatum, and consequently increase locomotor activity in male quail as in male newts.

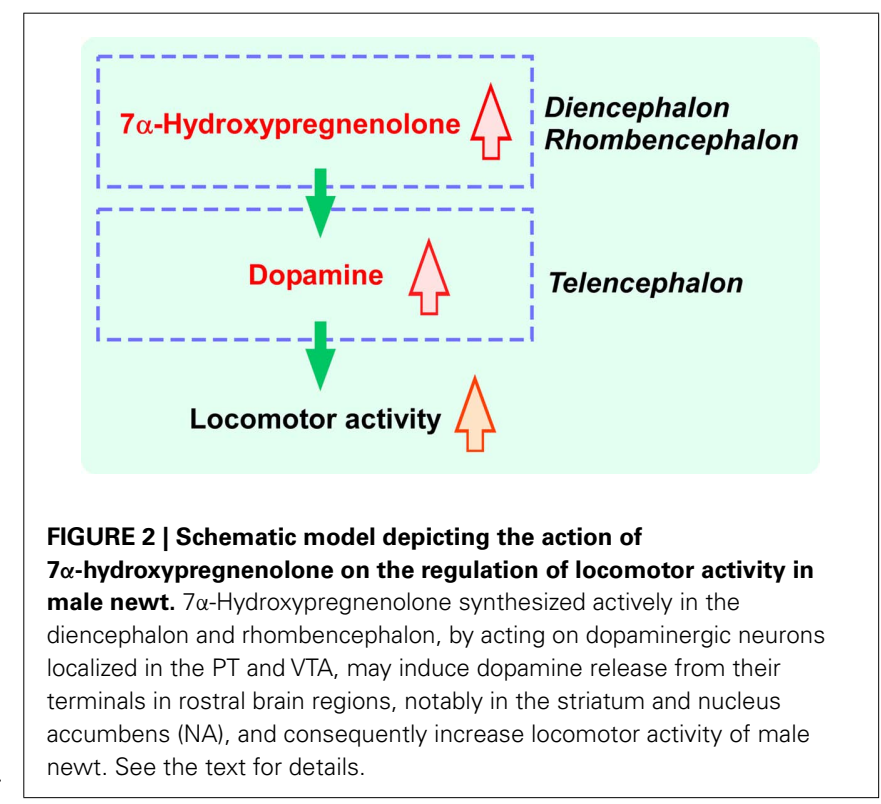

\section{$7 \alpha$-HYDROXYPREGNENOLONE ACTION THROUGH NON-GENOMIC MECHANISM}

The fact that $7 \alpha$-hydroxypregnenolone acutely increases locomotor activity in newts and quail suggests that this neurosteroid may act through a non-genomic rather than a genomic mechanism. It has been reported that in rats, the progesterone metabolite $3 \alpha, 5 \alpha-$ tetrahydroprogesterone ( $3 \alpha, 5 \alpha$-THP; allopregnanolone) exerts its effects on locomotion (Wieland et al., 1995) and dopamine release (Bullock et al., 1997; Rougé-Pont et al., 2002) via a nongenomic pathway. Allopregnanolone may act through modulation of $\mathrm{GABA}_{\mathrm{A}}$ receptors, since allopregnanolone is a potent allosteric modulator of $\mathrm{GABA}_{\mathrm{A}}$ receptors (Paul and Purdy, 1992; Lambert et al., 1995) and dopaminergic neurons are regulated by GABAergic transmission (Laviolette and van der Kooy, 2001). Whether the acute actions of $7 \alpha$-hydroxypregnenolone on dopamine release and locomotor activity in newts and quail are mediated through $\mathrm{GABA}_{\mathrm{A}}$ receptors remain to be determined.

\section{FUNCTIONAL SIGNIFICANCE OF $7 \alpha$-HYDROXYPREGNENOLONE}

\section{SEX-DEPENDENT $7 \alpha$-HYDROXYPREGNENOLONE SYNTHESIS AND ACTION}

It is well known that in vertebrates, locomotor activity of males is higher than that of females (Tsutsui, 1931; Iwata et al., 2000; Tsutsui et al., 2008). Sexually mature male newts in the breeding period move around much more than the females, searching sexually mature female partners or courting females prior to sperm transfer (Tsutsui, 1931; Iwata et al., 2000). In newts, the synthesis and concentration of $7 \alpha$-hydroxypregnenolone in the male brain were higher than in the female brain (Matsunaga et al., 2004; Haraguchi et al., 2010). Therefore, $7 \alpha$-hydroxypregnenolone may specifically affect the activity of male newts.

In quail, the production and concentration of $7 \alpha-$ hydroxypregnenolone in the male diencephalon were also higher than in the female diencephalon (Tsutsui et al., 2008). Such a sexual dimorphism only occurs in the diencephalon (Tsutsui et al., 
2008). In view of the sex difference in $7 \alpha$-hydroxypregnenolone synthesis and concentration in the quail diencephalon (Tsutsui et al., 2008), it is also possible that this neurosteroid actively plays a role in the control of locomotor activity in males (Tsutsui et al., 2008).

\section{DIURNAL CHANGES IN $7 \alpha$-HYDROXYPREGNENOLONE SYNTHESIS AND ACTION}

To investigate the functional significance of $7 \alpha$-hydroxypregnenolone in the regulation of locomotor activity, diurnal changes in both locomotor activity and diencephalic $7 \alpha$-hydroxypregnenolone concentrations were studied in male quail exposed to daily photoperiods of 16/8 h light/dark (LD; lights on at 07:00 a.m., off at 11:00 p.m.). Locomotor activity of males was much higher than that of females from the time of lights on until noon, but thereafter decreased to female levels (Tsutsui et al., 2008). In males, these changes in locomotor activity were correlated with concentrations of diencephalic $7 \alpha$-hydroxypregnenolone, the maximum value occurring at 11:00 a.m. when locomotor activity was high (Tsutsui et al., 2008). The functional significance of this correlation was supported by the observation that administration of ketoconazole, an inhibitor of P450s, inhibits locomotor activity at 11:00 a.m. (Tsutsui et al., 2008). Thus, the increase in diencephalic $7 \alpha$-hydroxypregnenolone may be responsible, at least in part, for the higher locomotor activity in males. As mentioned above, the low level of $7 \alpha$-hydroxypregnenolone synthesis and concentration in the female diencephalon suggests that this neurosteroid may not play a role in female locomotor activity.

\section{REGULATORY MECHANISMS OF DIURNAL CHANGES IN $7 \alpha$-HYDROXYPREGNENOLONE SYNTHESIS AND ACTION}

Melatonin is known to be also involved in the regulation of locomotor activity in birds (Binkley et al., 1971; John et al., 1978; Cassone and Menaker, 1984; Chabot and Menaker, 1992; Hau and Gwinner, 1994; Warren and Cassone, 1995; Murakami et al., 2001), which suggested that melatonin may regulate diencephalic $7 \alpha$-hydroxypregnenolone synthesis, and thereby influence locomotor activity. To elucidate the mechanism regulating diurnal changes in $7 \alpha$-hydroxypregnenolone synthesis and $7 \alpha$-hydroxypregnenolone-dependent locomotor activity, Tsutsui et al. (2008) performed a series of experiments involving melatonin manipulation in male quail. Combination of pinealectomy $(\mathrm{Px})$ and orbital enucleation (Ex) increased the production and concentration of $7 \alpha$-hydroxypregnenolone and the expression of cytochrome $\mathrm{P} 450_{7 \alpha}$ in the quail diencephalon after 1 week. Conversely, melatonin administration to $\mathrm{Px} / \mathrm{Ex}$ quail decreased the production and concentration of $7 \alpha$-hydroxypregnenolone and the expression of cytochrome $\mathrm{P} 450_{7 \alpha}$ in the diencephalon (Tsutsui et al., 2008). Further, the inhibitory effect of melatonin on $7 \alpha$-hydroxypregnenolone synthesis was abolished by luzindole, a melatonin receptor antagonist (Tsutsui et al., 2008). Melatonin derived from the pineal gland and eyes therefore may act as an inhibitory factor of $7 \alpha$-hydroxypregnenolone synthesis in the quail (Figure 3). This notion is supported by the earlier studies indicating that melatonin treatment decreases locomotor activity in quail (Murakami et al., 2001; Nakahara et al., 2003) and other birds (Murakami et al., 2001).

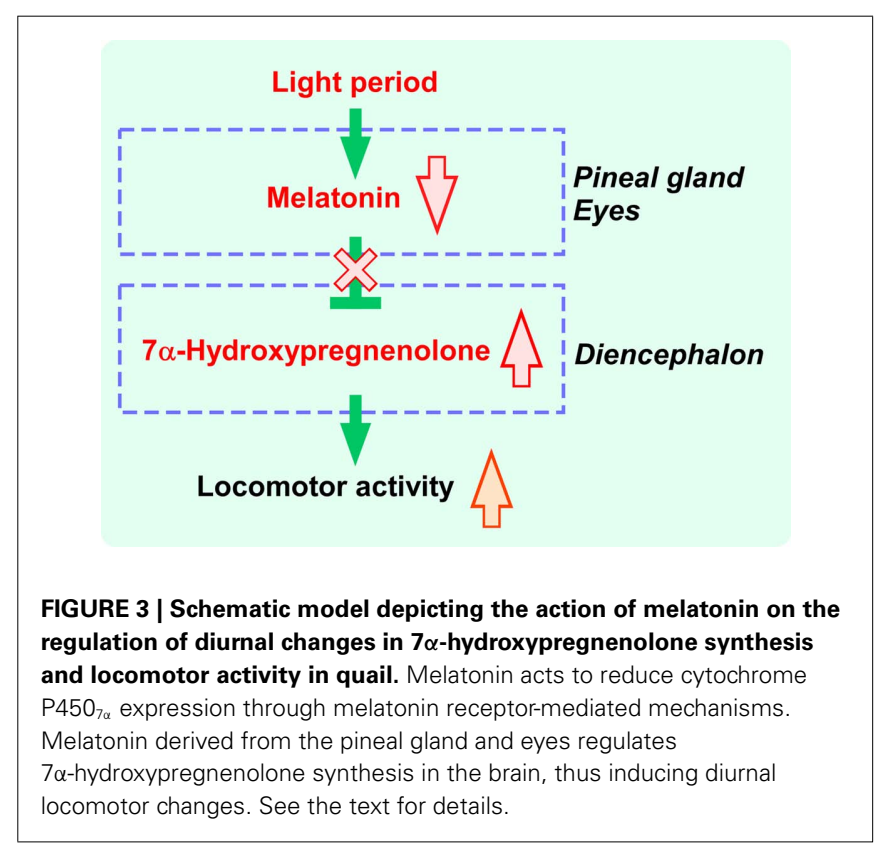

In quail, as in other vertebrates, the nocturnal secretion of melatonin is night-length dependent (Cockrem and Follett, 1985), and the onset of melatonin secretion occurs soon after the onset of darkness (Kumar and Follett, 1993). Therefore, the increase in $7 \alpha-$ hydroxypregnenolone synthesis in the brain of male quail during the light period is likely to be a result of the decrease in endogenous melatonin secretion (Figure 3). Since $7 \alpha$-hydroxypregnenolone stimulates locomotor activity, it is proposed that, in male quail, this neurosteroid plays a crucial role in diurnal changes in locomotor activity through the action of melatonin.

In birds and other vertebrates in general, locomotor activity undergoes a circadian rhythm (Saper et al., 2005) controlled by diurnal rhythm of melatonin secretion (Binkley et al., 1971; John et al., 1978; Cassone and Menaker, 1984; Chabot and Menaker, 1992; Hau and Gwinner, 1994; Warren and Cassone, 1995). However, the molecular mechanisms underlying this neurohormonal regulation of behavior have been poorly understood. The discovery of the role of $7 \alpha$-hydroxypregnenolone in mediating the action of melatonin on diurnal locomotor rhythmicity is an important step in understanding these mechanisms (Tsutsui et al., 2008). A similar mechanism may underly the regulation of diurnal locomotor rhythms in other vertebrates (for reviews, see Tsutsui et al., 2009a,b, 2010a,b), since $7 \alpha$-hydroxypregnenolone is also present in the brains of newts (Matsunaga et al., 2004) and mammals (Akwa et al., 1992; Doostzadeh and Morfin, 1997; Weill-Engerer et al., 2003; Yau et al., 2003).

\section{SEASONAL CHANGES IN $7 \alpha$-HYDROXYPREGNENOLONE SYNTHESIS AND ACTION}

To further understand the functional significance of $7 \alpha$-hydroxypregnenolone, seasonal changes in $7 \alpha$-hydroxypregnenolone synthesis and concentration in the brain were also demonstrated in newts (Matsunaga et al., 2004; Haraguchi et al., 2010). Both the synthesis and concentration of $7 \alpha$-hydroxypregnenolone in the male brain markedly changed during the annual breeding cycle 
and were maximum in the spring breeding period (Matsunaga et al., 2004; Haraguchi et al., 2009, 2010). Similar seasonal changes in the expression of cytochrome $\mathrm{P}^{4} 50_{7 \alpha}$ occurred in the male brain (Haraguchi et al., 2010). These findings suggest that the increase in locomotor activity of male newts in the spring breeding period can be accounted for an increase in $7 \alpha$-hydroxypregnenolone synthesis in the brain. In contrast to males, $7 \alpha$-hydroxypregnenolone levels in the brain of females did not vary significantly and are constantly low (Haraguchi et al., 2010). Accordingly, the lower locomotor activity in females could be ascribed to a lower level of $7 \alpha$-hydroxypregnenolone in their brain.

\section{REGULATORY MECHANISMS OF SEASONAL CHANGES IN 7 $\alpha$-HYDROXYPREGNENOLONE SYNTHESIS AND ACTION}

Plasma prolactin (PRL) levels in the male newt are elevated during the breeding period (Matsuda et al., 1990; Mosconi et al., 1994) and it has been shown that PRL acts directly on the brain to regulate courtship behavior in the male newt (Toyoda et al., 2005). Based on these observations, PRL may act on the brain to increase $7 \alpha$-hydroxypregnenolone synthesis, thus enhancing locomotor activity of male newts during the breeding period. A recent study has provided evidence that PRL is an important regulator of $7 \alpha$-hydroxypregnenolone production (Haraguchi et al., 2010; Figure 4). Hypophysectomy (Hypox) decreased $7 \alpha$-hydroxypregnenolone synthesis and concentration in the brain of sexually mature males after 2 weeks, suggesting that some pituitary hormone(s) may be involved in the regulation of $7 \alpha$-hydroxypregnenolone synthesis in the brain (Haraguchi et al., 2010). Administration of PRL but not gonadotropins (GTHs) to Hypox male newts caused a dose-dependent increase in $7 \alpha-$ hydroxypregnenolone synthesis and concentration in the brain (Haraguchi et al., 2010). Reciprocally, administration of anti-newt PRL serum dose-dependently decreased $7 \alpha$-hydroxypregnenolone biosynthesis (Haraguchi et al., 2010). Accordingly, PRL secreted by

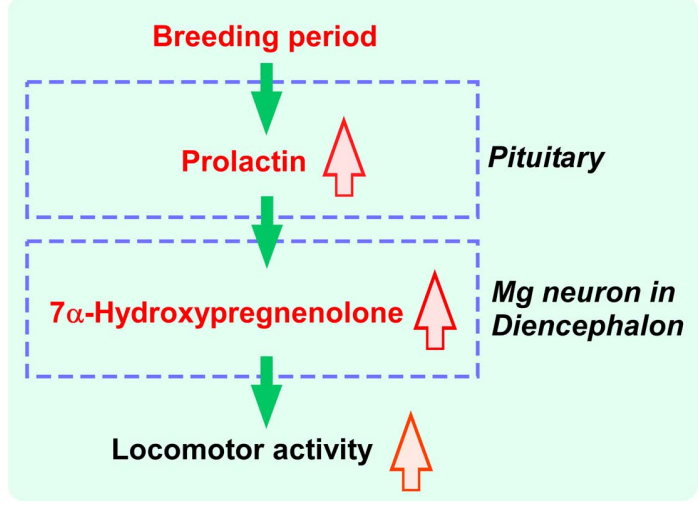

FIGURE 4 | Schematic model depicting the action of PRL on the regulation of seasonal changes in $7 \alpha$-hydroxypregnenolone synthesis and locomotor activity in newts. PRL synthesized in the adenohypophysis, by acting on Mg neurons in the hypothalamus, induces the expression of cytochrome $\mathrm{P}^{4} 50_{7 \alpha}$. Cytochrome $\mathrm{P}^{4} \mathrm{O}_{7 \alpha}$ and $\mathrm{PRL}$ receptor (PRLR) are colocalized in Mg neurons. Thus, prolactin, an adenohypophyseal hormone, regulates $7 \alpha$-hydroxypregnenolone synthesis in the brain, and also induces seasonal locomotor changes. See the text for details. the adenohypophysis can be regarded as a major factor regulating $7 \alpha$-hydroxypregnenolone synthesis (Figure 4). This is a previously undescribed role of the adenohypophyseal hormone in the regulation of neurosteroidogenesis in the brain in any vertebrate.

In contrast to male newts, no seasonal changes in $7 \alpha$-hydroxypregnenolone synthesis and concentration, and cytochrome $\mathrm{P} 450_{7 \alpha}$ mRNA expression were observed in female newts (Haraguchi et al., 2010). In newts, plasma PRL levels in males exhibit marked seasonal changes during the annual breeding cycle and are maximum in the spring breeding period (Matsuda et al., 1990; Mosconi et al., 1994). In contrast, plasma PRL levels in females are constantly low (Matsuda et al., 1990). Such a sex difference in the seasonal changes in plasma PRL levels may account for the absence of seasonal changes in $7 \alpha$-hydroxypregnenolone synthesis and concentration, and cytochrome $\mathrm{P}^{2} 50_{7 \alpha}$ mRNA expression in the female brain.

To understand the mode of action of PRL in the regulation of $7 \alpha$-hydroxypregnenolone synthesis, Haraguchi et al. (2010) determined the site of cytochrome $\mathrm{P} 450_{7 \alpha}$ expression and colocalization of cytochrome P450 $7 \alpha$ and PRL receptor (PRLR) in sexually mature male newts. P450 $0_{7 \alpha}$-positive cells were localized mainly in the anterior preoptic area (POA), magnocellular preoptic nucleus $(\mathrm{Mg})$, and tegmental area (TA) in the brain (Haraguchi et al., 2010). However, PRLR-like immunoreactivity was found only in the Mg (Haraguchi et al., 2010). Thus, the major, but perhaps not exclusive, targets of PRL action to increase $7 \alpha$-hydroxypregnenolone synthesis are the $\mathrm{P} 450_{7 \alpha}$-positive cells in the $\mathrm{Mg}$ (Figure 4). The Mg is sexually dimorphic both in terms of response to pheromones and neuroanatomical aspect (Govek and Swann, 2007). In particular, the $\mathrm{Mg}$ possesses more neurons in the male than in the female (Govek et al., 2003). Electrolytic lesions that include the Mg immediately and permanently eliminate male copulatory behavior in the hamster (Powers et al., 1987). In newt (Giorgio et al., 1982; Toyoda et al., 1993), the involvement of PRL in eliciting courtship behavior of males has been reported. Accordingly, it is possible that PRL may also induce the expression of locomotor activity and courtship behavior by increasing $7 \alpha$-hydroxypregnenolone synthesis in the $\mathrm{Mg}$ of sexually mature male newts (Figure 4).

On the other hand, it is known that in mammals, PRL is synthesized not only in the adenohypophysis but also in a subset of hypothalamic neurons projecting throughout the brain (Fuxe et al., 1977; De Vito, 1988; Emanuele et al., 1992). Based on the preliminary studies conducted by the laboratory of Kikuyam (I. Hasunuma and S. Kikuyama, unpublished observation), PRL was expressed in the newt brain but the expression level might be very low (see Haraguchi et al., 2010). Thus, the localization and function of brain PRL are still unclear in newts. It is considered that adenohypophyseal PRL is more important than brain PRL in the expressions of locomotor activity and courtship behavior, in as much as the increase in plasma PRL levels in breeding male newts (Matsuda et al., 1990; Mosconi et al., 1994) and the suppression of locomotor activity and courtship behavior in Hypox male newts (Toyoda et al., 1993; Haraguchi et al., 2010) have also been reported.

In mammals, choroid plexus PRLR has been proposed to be involved in the transport of PRL from blood into the cerebrospinal 
fluid (Walsh et al., 1987). In the choroid plexus of newts, dense PRLR immunoreactivity and PRLR mRNA signals were observed in the epithelial cells (Hasunuma et al., 2005). Thus, PRL transported from the blood into the cerebrospinal fluid via the choroid plexus receptor is considered to play an important role in the expression of locomotor activity and courtship behavior, although a possible contribution of PRL transported to the brain through retrograde blood flow by the portal system cannot be excluded as reported in mammals (Oliver et al., 1977; Porter et al., 1978).

\section{EFFECTS OF STRESS ON $7 \alpha$-HYDROXYPREGNENOLONE SYNTHESIS AND ACTION}

It is firmly established that locomotor activity of vertebrates changes after acute stress (Lee et al., 1986; Lowry et al., 2009; Hubbard et al., 2010). Numerous studies in various vertebrates document that concentrations of adrenal steroid hormones, namely cortisol or corticosterone, increase shortly after exposure to stressful conditions (Coddington et al., 2007; Kirby et al., 2009). There is also evidence that injection of corticosterone rapidly and dramatically changes male locomotor activity (Moore and Miller, 1984; Mitra and Sapolsky, 2008; Ricciardella et al., 2010). However, the molecular mechanisms involved in corticosterone regulation of behavioral changes under stress are still obscure.

Based on these observations, we hypothesized that acute stress may increase $7 \alpha$-hydroxypregnenolone synthesis via corticosterone action in the newt brain, and that $7 \alpha$-hydroxypregnenolone may subsequently increase locomotor activity. To test these hypotheses, we conducted a series of experiments using the male newt (S. Haraguchi, T. Koyama, S. I. Hasunuma, S. Okuyama, S. Kikuyama, J. L. Do-Rego, H. Vaudry, and K. Tsutsui, unpublished observation). A 30-min restraint stress increased $7 \alpha-$ hydroxypregnenolone synthesis and plasma corticosterone levels in male newts. Hypox decreased $7 \alpha$-hydroxypregnenolone synthesis, whereas administration of corticosterone to Hypox newts caused an increase in $7 \alpha$-hydroxypregnenolone synthesis. These

\section{REFERENCES}

Akwa, Y., Morfin, R. F., Robel, P., and Baulieu, E.-E. (1992). Neurosteroid metabolism: $\quad 7 \alpha$-Hydroxylation of dehydroepiandrosterone and pregnenolone by rat brain microsomes. Biochem. J. 288, 959-964.

Ball, G. F., Casto, J. M., and Balthazart, J. (1995). Autoradiographic localization of D1-like dopamine receptors in the forebrain of male and female Japanese quail and their relationship with immunoreactive tyrosine hydroxylase. J. Chem. Neuroanat. 9, 121-133.

Bardo, M. T., Bowling, S. L., and Pierce, R. C. (1990). Changes in locomotion and dopamine neurotransmission following amphetamine, haloperidol, and exposure to novel environmental stimuli. Psychopharmacology 101, 338-343.

Baulieu, E.-E. (1997). Neurosteroids: of the nervous system, by the nervous system, for the nervous system. Recent Prog. Horm. Res. 52, $1-32$.

Beaujean, D., Mensah-Nyagan, A. G., Do-Rego, J. L., Luu-The, V., Pelletier, G., and Vaudry, H. (1999). Immunocytochemical localization and biological activity of hydroxysteroid sulfotransferase in the frog brain. J. Neurochem. 72, 848-857.

Binkley, S., Kluth, E., and Menaker, M. (1971). Pineal function in sparrows: circadian rhythms and body temperature. Science 174, 311-314.

Bruzzone, F., Do-Rego, J. L., LuuThe, V., Pelletier, G., Vallarino, M., and Vaudry, H. (2010). Immunohistochemical localization

results provide new evidence that $7 \alpha$-hydroxypregnenolone, a key neurosteroid implicated in the induction of locomotion, mediates the action of corticosterone to modulate locomotor activity in newts under stress.

\section{CONCLUSION AND FUTURE DIRECTIONS}

In conclusion, $7 \alpha$-hydroxypregnenolone, a newly discovered amphibian and avian neurosteroid, acts as an important factor stimulating locomotor activity. The stimulatory action of $7 \alpha-$ hydroxypregnenolone is mediated by the dopaminergic system. $7 \alpha$-Hydroxypregnenolone apparently functions in males but not in females. Melatonin acts on the neurons expressing cytochrome $\mathrm{P} 450_{7 \alpha}$ to regulate $7 \alpha$-hydroxypregnenolone synthesis, thus inducing diurnal locomotor changes. PRL, an adenohypophyseal hormone, also acts on the neurons expressing cytochrome $\mathrm{P} 450_{7 \alpha}$ to regulate $7 \alpha$-hydroxypregnenolone synthesis, thus inducing seasonal locomotor changes. $7 \alpha$-Hydroxypregnenolone further mediates the action of corticosterone to modulate locomotor activity under stress.

The synthesis of $7 \alpha$-hydroxypregnenolone increases during the breeding season and decreases during the non-breeding season. These seasonal changes suggest that $7 \alpha$-hydroxypregnenolone may be involved in maintaining energy balance via energy conservation during lean times. Future study is needed to demonstrate this hypothesis. In addition, various wild animals migrate just before reproduction. They become very active at the time of migration. $7 \alpha$-Hydroxypregnenolone may also drive animals to migrate and enhance migratory activity. Avian migration is a good model to demonstrate this hypothesis.

\section{ACKNOWLEDGMENTS}

The authors thank Kazuhiko Inoue, Hitomi Miyabara, Saori Suzuki, Teppei Koyama, Takayoshi Ubuka, Itaru Hasunuma, Sakae Kikuyama, and Jean-Luc Do-Rego for their work cited in this manuscript. This work was supported by Grants-in-Aid for Scientific Research from the Ministry of Education, Science and Culture, Japan (18107002, 22132004, and 22227002 to Kazuyoshi Tsutsui).

and biological activity of $3 \beta$ hydroxysteroid dehydrogenase and $5 \alpha$-reductase in the brain of the frog, Rana esculenta, during development. J. Chem. Neuroanat. 39, 35-50.

Bullock, A. E., Clark, A. L., Grady, S. R., Robinson, S. F., Slobe, B. S., Marks, M. J., and Collins, A. C. (1997). Neurosteroids modulate nicotinic receptor function in mouse striatal and thalamic synaptosomes. J. Neurochem. 68, 2412-2423.

Cassone, V. M., and Menaker, M. (1984). Is the avian circadian system a neuroendocrine loop? J. Exp. Zool. 232, 539-549.

Chabot, C. C., and Menaker, M. (1992). Circadian feeding and locomotor rhythms in pigeons and house sparrows. J. Biol. Rhythms 7, 287-299.
Cockrem, J. F., and Follett, B. K (1985). Circadian rhythm of melatonin in the pineal gland of the Japanese quail (Coturnix coturnix japonica). J. Endocrinol. 107, 317-324.

Coddington, E., Lewis, C., Rose, J. D., and Moore, F. L. (2007). Endocannabinoids mediate the effects of acute stress and corticosterone on sex behavior. Endocrinology 148, 493-500.

Compagnone, N. A., and Mellon, S. H. (2000). Neurosteroids: biosynthesis and function of these novel neuromodulators. Front. Neuroendocrinol. 21:1-56. doi: 10.1006/frne.1999.0188

De Vito, W. J. (1988). Distribution of immunoreactive prolactin in the male and female rat brain: effects of hypophysectomy and 
intraventricular administration of colchicines. Neuroendocrinology 47, 284-289.

Doostzadeh, J., and Morfin, R. (1997). Effects of cytochrome P450 inhibitors and of steroid hormones on the formation of 7-hydroxylated metabolites of pregnenolone in mouse brain microsomes. J. Endocrinol. 155, 343-350.

Do-Rego, J. L., Seong, J. Y., Burel, D., Leprince, J., Luu-The, V., Tsutsui, K., Tonon, M.-C., Pelletier, G., and Vaudry, H. (2009). Neurosteroid biosynthesis: enzymatic pathways and neuroendocrine regulation by neurotransmitters and neuropeptides. Front. Neuroendocrinol. 30, 259-301.

Do-Rego, J. L., Tremblay, Y., Luu-The, V., Repello, E., Vallarino, M., Belanger, A., Pelletier, G., and Vaudry, H. (2007). Immunocytochemical localization and biological activity of the steroidogenic enzyme cytochrome P450 17 $\alpha$-hydroxylase/C17, 20lyase $\left(\mathrm{P}_{450} \mathrm{C} 17\right)$ in the frog brain and pituitary. J. Neurochem. 100, 251-268.

Emanuele, N. V., Jurgens, J. K., Halloran, M. M., Tentler, J. J., Lawrence, A. M., and Kelley, M. R. (1992). The rat prolactin gene is expressed in brain tissue: detection of normal and alternative spliced prolactin messenger RNA. Mol. Endocrinol. 6, 35-42.

Fuxe, K., Hokfelt, T., Eneroth, P., Gustafsson, J. A., and Skett, P. (1977). Prolactin-like immunoreactivity: localization in nerve terminals of rat hypothalamus. Science 196, 899-900.

Giorgio, M., Giacoma, C., Vellano, C., and Mazzi, V. (1982). Prolactin and sexual behavior in the crested newt (Triturus cristatus carnifex Laur). Gen. Comp. Endocrinol. 47, 139-147.

Govek, E. K., and Swann, J. M. (2007). Stereological sex difference during development of the magnocelluar subdivision of the medial preoptic nucleus (MPN mag). Brain Res. 1145, 90-96.

Govek, E. K., Wang, J., and Swann, J. M. (2003). Sex differences in the magnocellular subdivision of the medial preoptic nucleus in Syrian hamsters. Neuroscience 116, 593-598.

Hara, E., Kubikova, L., Hessler, N. A., and Jarvis, E. D. (2007). Role of the midbrain dopaminergic system in modulation of vocal brain activation by social context. Eur. J. Neurosci. 25, 3406-3416.

Haraguchi, S., Koyama, T., Hasunuma, I., Vaudry, H., and Tsutsui, K. (2010). Prolactin increases the synthesis of $7 \alpha$-hydroxypregnenolone, a key factor for induction of locomotor activity, in breeding male newts. Endocrinology 151, 2211-2222.

Haraguchi, S., Matsunaga, M., Koyama, T., Do Rego, J. L., and Tsutsui, K. (2009). Seasonal changes in the synthesis of the neurosteroid $7 \alpha$-hydroxypregnenolone stimulating locomotor activity in newts. Ann. N. Y. Acad. Sci. 1163, 410-413.

Hasunuma, I., Toyoda, F., Yamamoto, K., Yamashita, M., and Kikuyama, S. (2005). Localization of prolactin receptor in the newt brain. Cell Tissue Res. 320, 477-485.

Hau, M., and Gwinner, E. (1994). Melatonin facilitates synchronization of sparrow circadian rhythms to light. J. Comp. Physiol. A 175, 343-347.

Hubbard, C. S., Dolence, E. K., and Rose, J. D. (2010). Brainstem reticulospinal neurons are targets for corticotropin-releasing factorInduced locomotion in roughskin newts. Horm. Behav. 57, 237-546.

Inai, Y., Nagai, K., Ukena, K., Oishi, T., and Tsutsui, K. (2003). Seasonal changes in neurosteroids in the urodele brain and environmental factors inducing their changes. Brain Res. 959, 214-225.

Iwata, T., Toyoda, F., Yamamoto, K., and Kikuyama, S. (2000). Hormonal control of urodele reproductive behavior. Comp. Biochem. Physiol. B Biochem. Mol. Biol. 126, 221-229.

John, T. M., Itoh, S., and George, J. C. (1978). On the role of the pineal in thermoregulation in the pigeon. Horm. Res. 9, 41-56.

Kirby, E. D., Geraghty, A. C., Ubuka, T., Bentley, G. E., and Kaufer, D. (2009). Stress increases putative gonadotropin inhibitory hormone and decreases luteinizing hormone in male rats. Proc. Natl. Acad. Sci. U.S.A. 106, 11324-11329.

Kumar, V., and Follett, B. K. (1993). The circadian nature of melatonin secretion in Japanese quail (Coturnix coturnix japonica). J. Pineal Res. 14, 192-200.

Lambert, J. J., Belelli, D., Hill-Venning, C., and Peters, J. A. (1995). Neurosteroids and $\mathrm{GABA}_{\mathrm{A}}$ receptor function. Trends Pharmacol. Sci. 16, 295-303.

Laviolette, S. R., and van der Kooy, D. (2001). $\mathrm{GABA}_{\mathrm{A}}$ receptors in the ventral tegmental area control bidirectional reward signalling between dopaminergic and nondopaminergic neural motivational systems. Eur. J. Neurosci. 13, 1009-1015.

Lee, E. H., Tsai, M. J., and Chai, C. Y. (1986). Stress selectively influences center region activity of mice in an open field. Physiol. Behav. 37, 659-662.

Levens, N., Green, T. A., Akins, C. K. and Bardo, M. T. (2000). Dopamine $\mathrm{D}_{2}$-like receptor binding in the brain of male Japanese quail (Coturnix japonica). Neurosci. Lett. 22, 77-80.

Lowry, C. A., Burke, K. A., Renner, K. J., Moore, F. L., and Orchinik, M. (2001). Rapid changes in monoamine levels following administration of corticotropin-releasing factor or corticosterone are localized in the dorsomedial hypothalamus. Horm. Behav. 39, 195-205.

Lowry, C. A., Hale, M. W., Burke, K. A., Renner, K. J., and Moore, F. L. (2009). Fluoxetine potentiates the effects of corticotropin-releasing factor on locomotor activity and serotonergic systems in the roughskin newt, Taricha granulosa. Horm. Behav. 56 177-184.

Matsuda, K., Tanaka, S., Yamamoto, K., and Kikuyama, S. (1990). Annual changes of plasma prolactin levels in the newt, Cynops pyrrhogaster. Zool. Sci. 7, 1143 .

Matsunaga, M., Ukena, K., Baulieu, E.-E., and Tsutsui, K. (2004). $7 \alpha-$ Hydroxypregnenolone acts as a neuronal activator to stimulate locomotor activity of breeding newts by means of the dopaminergic system. Proc. Natl. Acad. Sci. U.S.A. 101, 17282-17287.

Mellon, S. H., and Vaudry, H. (2001). Biosynthesis of neurosteroids and regulation of their synthesis. Int. Rev. Neurobiol. 46, 33-78.

Mensah-Nyagan, A. G., Do-Rego, J. L., Beaujean, D., Luu-The, V., Pelletier, G., and Vaudry, H. (1999). Neurosteroids: expression of steroidogenic enzymes and regulation of steroid biosynthesis in the central nervous system. Pharmacol. Rev. 51, 63-81.

Mensah-Nyagan, A. G., Do-Rego, J. L., Feuilloley, M., Marcual, A., Lange, C., Pelletier, G., and Vaudry, H. (1996a). In vivo and in vitro evidence for the biosynthesis of testosterone in the telencephalon of the female frog. $J$. Neurochem. 67, 413-422.

Mensah-Nyagan, A. G., Feuilloley, M., Do-Rego, J. L., Marcual, A., Lange, C., Tonon, M. C., Pelletier, G., and Vaudry, H. (1996b). Localization of $17 \alpha$-hydroxysteroid dehydrogenase and characterization of testosterone in the brain of the male frog. Proc. Natl. Acad. Sci. U.S.A. 93, 1423-1428.

Mensah-Nyagan, A. G., Feuilloley, M., Dupont, E., Do-Rego, J. L., Leboulenger, F., Pelletier, G., and Vaudry, H. (1994). Immunocytochemical localization and biological activity of $3 \beta$-hydroxysteroid dehydrogenase in the central nervous system of the frog. J. Neurosci. 14, 7306-7318.

Mezey, S., and Csillag, A. (2002). Selective striatal connections of midbrain dopaminergic nuclei in the chick (Gallus domesticus). Cell Tissue Res. 308, 35-46.

Mitra, R., and Sapolsky, R. M. (2008). Acute corticosterone treatment is sufficient to induce anxiety and amygdaloid dendritic hypertrophy. Proc. Natl. Acad. Sci. U.S.A. 105, 5573-5578.

Moore, F. L., and Miller, L. J. (1984). Stress-induced inhibition of sexual behavior: corticosterone inhibits courtship behaviors of a male amphibian (Taricha granulosa). Horm. Behav. 18, 400-410.

Mosconi, G., Yamamoto, K., Kikuyama, S., Carnevali, O., Mancuso, A., and Vellano, C. (1994). Seasonal changes of plasma prolactin concentration in the reproduction of the crested newt (Triturus carnifex Laur). Gen. Comp. Endocrinol. 95, 342-349.

Murakami, N., Kawano, T., Nakahara, K., Nasu, T., and Shiota, K. (2001). Effect of melatonin on circadian rhythm, locomotor activity and body temperature in the intact house sparrow, Japanese quail and owl. Brain Res. 889, 220-224.

Nakahara, K., Kawano, T., Shiota, K., and Murakami, N. (2003). Effects of microinjection of melatonin into various brain regions of Japanese quail on locomotor activity and body temperature. Neurosci. Lett. 345, 117-120.

Oliver, C., Mical, R. S., and Porter, J. C. (1977). Hypothalamic-pituitary vasculature: evidence for retrograde blood flow in the pituitary stalk. Endocrinology 101, 598-604.

Paul, S. M., and Purdy, R. H. (1992). Neuroactive steroids. FASEB J. 6 , 2311-2322.

Porter, J. C., Barnea, A., Cramer, O. M. and Parker, C. R. Jr. (1978). Hypothalamic peptide and catecholamine secretion: roles for portal and retrograde blood flow in the pituitary stalk in the release of hypothalamic dopamine and pituitary prolactin and LH. Clin. Obstet. Gynaecol. 5, 271-282.

Powers, J. B., Newman, S. W., and Bergondy, M. L. (1987). MPOA and BNST lesions in male Syrian hamsters: differential effects on copulatory and chemoinvestigatory behaviors. Behav. Brain Res. 23, 181-195.

Ricciardella, L. F., Bliley, J. M., Feth, C. C., and Woodley, S. K. (2010). Acute stressors increase plasma corticosterone and decrease locomotor 
activity in a terrestrial salamander (Desmognathus ochrophaeus). Physiol. Behav. 101, 81-86.

Rougé-Pont, F., Mayo, W., Marinelli, M., Gingras, M., Moal, M. L., and Piazza, P. V. (2002). The neurosteroid allopregnanolone increases dopamine release and dopaminergic response to morphine in the rat nucleus accumbens. Eur. J. Neurosci. $16,169-173$

Sanberg, P. R. (1983). Dopaminergic and cholinergic influences on motor behavior in chickens. J. Comp. Psychol. 97, 59-68.

Saper, C. B., Lu, J., Chou, T. C., and Gooley, J. (2005). The hypothalamic integrator for circadian rhythms. Trends Neurosci. 28, 152-157.

Sharp, T., Zetterström, T., Ljungberg, T., and Ungerstedt, U. (1987). A direct comparison of amphetamineinduced behaviours and regional brain dopamine release in the rat using intracerebral dialysis. Brain Res. 401, 322-330.

Takase, M., Haraguchi, S., Hasunuma, I., Kikuyama, S., and Tsutsui, K. (2011). Expression of cytochrome P450 side-chain cleavage enzyme mRNA in the brain of the red-bellied newt Cynops pyrrhogaster. Gen. Comp. Endocrinol. 170, 468-474.

Takase, M., Ukena, K., and Tsutsui, K. (2002). Expression and localization of cytochrome P45011 $\beta$, aldo mRNA in the frog brain. Brain Res. 950, 288-296.

Takase, M., Ukena, K., Yamazaki, T., Kominami, S., and Tsutsui, K. (1999). Pregnenolone, pregnenolone sulfate and cytochrome P450 side-chain cleavage enzyme in the amphibian brain and their seasonal changes. Endocrinology 140, 1936-1944.

Toyoda, F., Hasunuma, I., Yamamoto, K., Yamashita, M., and Kikuyama, S. (2005). Prolactin acts centrally to enhance newt courtship behavior. Gen. Comp. Endocrinol. 141, 172-177.

Toyoda, F., Ito, M., Tanaka, S., and Kikuyama, S. (1993). Hormonal induction of male courtship behavior in the Japanese newt, Cynops pyrrhogaster. Horm. Behav. 27, 511-522.

Tsutsui, K., Haraguchi, S., Inoue, K., Miyabara, H., Suzuki, S., Ogura, Y., Koyama, T., Matsunaga, M., and Vaudry, H. (2009a). Identification, biosynthesis, and function of $7 \alpha-$ hydroxypregnenolone, a new key neurosteroid controlling locomotor activity, in nonmammalian vertebrates. Ann. N. Y. Acad. Sci. 1163 , 308-315.

Tsutsui, K., Inoue, K., Miyabara, H., Suzuki, S., Ogura, Y., Tobari, Y., and Haraguchi, S. (2009b). Discovery of a novel avian neurosteroid, $7 \alpha$ hydroxypregnenolone, and its role in the regulation of the diurnal rhythm of locomotor activity in Japanese quail. Gen. Comp. Endocrinol. 163, 117-122.

Tsutsui, K., Haraguchi, S., Matsunaga, M., Inoue, K., and Vaudry, $\mathrm{H}$. (2010a). 7 $\alpha$-Hydroxypregnenolone, a new key regulator of locomotor activity of vertebrates: identification, mode of action and functional significance. Front. Endocrinol. 1:9. doi: 10.3389/fendo.2010.00009

Tsutsui, K., Haraguchi, S., Matsunaga, M., Koyama, T., Do-Rego, J. L., and Vaudry, H. (2010b). Identification of $7 \alpha$-hydroxypregnenolone, a novel bioactive amphibian neurosteroid stimulating locomotor activity, and its physiological roles in the regulation of locomotion. Gen. Comp. Endocrinol. 168, 275-279.

Tsutsui, K., Inoue, K., Miyabara, H., Suzuki, S., Ogura, Y., and Haraguchi, S. (2008). $7 \alpha$-Hydroxypregnenolone mediates melatonin action underlying diurnal locomotor rhythms. $J$. Neurosci. 28, 2158-2167.

Tsutsui, K., Matsunaga, M., Miyabara, H., and Ukena, K. (2006). Neurosteroid biosynthesis in the quail brain. J. Exp. Zool. 305A, 733-742.

Tsutsui, K., Matsunaga, M., and Ukena, K. (2003). Biosynthesis and biological actions of neurosteroids in the avian brain. Avian Poult. Biol. Rev. 14, 63-78.

Tsutsui, K., and Mellon, S. H. (2006). Neurosteroids in the brain neuron: biosynthesis, action and medicinal impact on neurodegenerative disease. Central Nerv. Syst. Agents Med. Chem. 6, 73-82.

Tsutsui, K., Ukena, K., Takase, M. Kohchi, C., and Lea, R. W. (1999). Neurosteroid biosynthesis in vertebrate brains. Comp. Biochem. Physiol. C Toxicol. Pharmacol. 124, 121-129.

Tsutsui, K., Ukena, K., Usui, M., Sakamoto, H., and Takase, M. (2000). Novel brain function: biosynthesis and actions of neurosteroids in neurons. Neurosci. Res. 36 261-273.

Tsutsui, Y. (1931). Notes on the behavior of the common Japanese newt, Diemyctylus pyrrhogaster BOIE. I. Breeding habit. Mem. Col. Sci. Kyoto Imp. Univ. Ser. B7, 159-179.

Wada, M. (1979). Photoperiodic control of LH secretion in Japanese quail with special reference to the photoinducible phase. Gen. Comp. Endocrinol. 39, 141-149.

Walsh, R. G., Slaby, F. J., and Posner, B. I. (1987). A receptormediated mechanism for the transport of prolactin from blood to cerebrospinal fluid. Endocrinology 120, 1846-1850.

Warren, W. S., and Cassone, V. M. (1995). The pineal gland: photoreception and coupling of behavioral, metabolic, and cardiovascular circadian outputs. J. Biol. Rhythms 10, 64-79.

Weill-Engerer, S., David, J. P., Sazdovitch, V., Liere, P., Schumacher, M., Delacourte, A., Baulieu, E.-E., and Akwa, Y. (2003). In vitro metabolism of dehydroepiandrosterone (DHEA) to $7 \alpha$-hydroxy-DHEA and $\Delta^{5}$

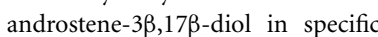
regions of the aging brain from Alzheimer's and non-demented patients. Brain Res. 969, 117-125.

Wieland, S., Belluzzi, J. D., Stein, L., and Lan, N. C. (1995). Comparative behavioral characterization of the neuroactive steroids 3 alpha$\mathrm{OH}, 5$ alpha-pregnan-20-one and 3 alpha-OH, 5 beta-pregnan-20-one in rodents. Psychopharmacology 118 , 65-71.

Wilson, W. O. (1972). A review of the physiology of Coturnix (Japanese quail). World's Poult. Sci. J. 28, 413-429.

Yau, J. L., Rasmuson, S., Andrew, R., Graham, M., Noble, J., Olsson, T., Fuchs, E., Lathe, R., and Seckl, J. R. (2003). Dehydroepiandrosterone 7-hydroxylase CYP7B: predominant expression in primate hippocampus and reduced expression in Alzheimer's disease. Neuroscience $121,307-314$

Conflict of Interest Statement: The authors declare that the research was conducted in the absence of any commercial or financial relationships that could be construed as a potential conflict of interest.

Received: 14 May 2011; paper pending published: 29 June 2011; accepted: 04 August 2011; published online: 22 August 2011.

Citation: Haraguchi S, Matsunaga M, Vaudry H and Tsutsui K (2011) Mode of action and functional significance of $7 \alpha-$ hydroxypregnenolone stimulating locomotor activity. Front. Endocrin. 2:23. doi: 10.3389/fendo.2011.00023

This article was submitted to Frontiers in Neuroendocrine Science, a specialty of Frontiers in Endocrinology.

Copyright (c) 2011 Haraguchi, Matsunaga, Vaudry and Tsutsui. This is an open-access article subject to a nonexclusive license between the authors and Frontiers Media SA, which permits use, distribution and reproduction in other forums, provided the original authors and source are credited and other Frontiers conditions are complied with. 